\title{
Die Vorbereitung der slowakischen Industriebetriebe auf den Beitritt in die Europäische Union (Research Note)*
}

\author{
Miroslav Majtán, Jana Sršňová ${ }^{* *}$
}

The paper defines crucial adjustment instruments of the Slovak business sector in the course of its accession to the European Union. Analysis of these instruments is illustrated with a sample of 110 Slovak manufacturing firms. Analysis shows that companies with over 500 employees, in particular in engineering and electrical engineering industries are better prepared for the accession. These companies are distinguish themselves with a high level of internationalization (a high share of exports in the EU market on the company's sales), utilization of higher forms of input on the common EU market, utilization of 9000 ISO standards and participation of a foreign investor, especially from the European Union. At the same time, analysis pointed to several risks, namely those related to competitiveness. Business in the size group from 50 to 499 employees with a higher rate of added value have to be paid a greater attention.

Der Aufsatz definiert wichtige Anpassungsinstrumente des slowakischen Wirtschaftsbereichs während des Beitritts in die Europäische Union. Die Analyse dieser Instrumente wird anhand einer Umfrage unter 100 slowakischen Produktionsbetrieben dargestellt. Die Analyse zeigt, dass Firmen mit mehr als 500 Beschäftigten besser auf den Beitritt vorbereitet ist. Diese Unternehmen zeichnen sich durch eine hohe Internationalisierung (ein grosser Anteil von Exporten in den EU-Markt an den Umsatzerlösen), Gebrauch von 9000 ISO Standards und die Beteiligung ausländischer Investoren auf, insbesondere von der Europäischen Union. Zur gleichen Zeit zeigte die Analyse mehrere Risiken auf, insbesondere in der Wettbewerbsfähigkeit Firmen in der Größenordnung von 50 bis 499 Angestellten mit einer höheren Rate von Mehrwert müssen stärker berücksichtigt werden.

Key words: Slovakia / European Union / internalization / competitiveness

\footnotetext{
Manuscript received: 17.10.2002, accepted: 17.03.2004 (2 revisions)

** Miroslav Majtán, Professor, Faculty of Management, University of Economics Bratislava. Corresponding address: mikusinc@dec.euba.sk

EDITORIAL REMARK: Since issue 1/2004 JEEMS only publishes papers and research notes in English. We make an exception here, because this paper was already submitted for the first time in 2002 !
} 
Mit der EU Osterweiterung entstanden zahlreiche Diskussionen über die Frage, inwieweit die beitretenden Staaten auf ihre EU- Mitgliedschaft vorbereitet sind. Die meisten dieser Diskussionen werden im Bereich der Wirtschaft geführt. Im Zuge des Beitrittes der SR zur EU wurden daher mehrere Studien erarbeitet, welche den Stand der Vorbereitung beurteilen. In der SR sind derzeit drei Studien bekannt: die Studie des Wirtschaftsinstitutes der Slowakischen Akademie der Wissenschaften, die Studie der Handelsfakultät der Ökonomischen Universität und die Studie des Konservativen Instituts. Alle drei Studien betrachten die Auswirkungen des Beitritts anhand makroökonomischer Größen wie Wirtschaftswachstum, Arbeitslosigkeit, Außenhandelsbilanz, Zufluss ausländischer Investitionen, Steigerung/ Senkung der nominalen und realen Löhne, Auswirkungen der Preise usw.

Die Analyse, deren Ergebnisse im folgenden präsentiert werden, ist ein Teil der Studie der Handelsfakultät der Ökonomischen Universität. Ihre Besonderheit besteht in der betriebswirtschaftlichen Ausrichtung mit dem Ziel die Vorbereitung der Industriebetriebe der SR auf den EU Beitritt zu untersuchen.

Die Analyse geht dabei von der Beurteilung der für die betriebliche Sphäre notwendigen Instrumente aus. Diese können in drei Gruppen unterteilt werden:

A. Ausgewählte Charakteristik, die mit der Internationalisierung des Unternehmens zusammenhängt - Anteil des Exports am Erlös des Betriebes, Anteil des Exports in die EU am Betriebserlös, Anteil des Exports in die Länder der V4 (die Visegrad-Länder) am Erlös der Unternehmen, sowie angewendete Beitrittsformen zum Markt der EU.

In der Praxis zeigt sich, dass das Maß der Internationalisierung der Betriebe (Anteil des Exports am Erlös), ihre Orientierung an den EU- Ländern, sowie die Form des Eintritts auf die EU Märkte unmittelbar die Vorbereitung der Industriebetriebe beeinflussen. Weiterhin gelten ein hoher Anteil am Export, die territoriale Ausrichtung auf die EU - Länder, sowie der Einsatz direkter Formen der Etablierung auf dem Auslandsmarkt, die auch die Marktpräsenz des entsprechenden Marktes ermöglichen (z.B. Vertriebsniederlassungen im Ausland) als bestimmend im Vorbereitungsprozess.

B. Eine besonders wichtige Rolle im Beitrittsprozess spielt die Konkurrenzfähigkeit der slowakischen Industriebetriebe. Gegenstand der Analyse sind deshalb die internen und externen Faktoren, welche sich mit der Unternehmenstätigkeit auf den EU-Märkten (Motive, Stärken und Schwächen auf dem Inlands- und EU-Markt, Informationsquellen, Einführung der ISO Normen, Ausnutzung der Strategie-, und Exportunterstützung auf dem EUMarkt), der Auswertung des Einflusses des gemeinsamen EU-Marktes auf die Unternehmen, und Erwartungen der Unternehmen (Ertrags- und Wachstumserwartungen, Chancen und Risiken) bei künftiger Mitwirkung auf dem EU-Markt, beschäftigen. 
C. Für die strategische Entwicklung der slowakischen Industriebetriebe sind Auslandsinvestoren von großer Bedeutung. Deshalb ist der Einstieg von Auslandsinvestoren, die den slowakischen Betrieben bei der Adaption an den EU Markt helfen (insbesondere aus der EU stammende Investoren), die Teilnahme der Auslandsinvestoren in slowakischen Industrieunternehmen, deren Beiträge für das Unternehmen, das Herkunftsland der strategischen Investoren, die Staatsunterstützung sowie die Erfüllung der Erwartungen des strategischen Investors, zu betrachten.

Der Beitrag ist in drei Teile gegliedert:

1. Charakteristik des Untersuchungsobjektes

2. Ergebnisse der durchgeführten Umfrage

3. Schlussfolgerung der durchgeführten Umfrage und Verallgemeinerung der Untersuchungsergebnisse

\section{Charakteristik des Untersuchungsobjektes}

Die Umfrage betrifft die Industriebetriebe der SR. Die Stellung des Industriebetriebes kennzeichnet die Verteilung des BIP-s nach Wirtschaftszweigen. (Tab. 1)

Tabelle 1. Gross domestic product by economic activists in $2000 \mathrm{in} \%$

\begin{tabular}{|l|c|}
\hline Total & $\mathbf{1 0 0} \%$ \\
\hline Market Goods & 35,7 \\
Industrie total & 26,4 \\
Market Services & 42,1 \\
Non Market Services & 12,5 \\
Other & 9,7 \\
\hline
\end{tabular}

Quelle: Štatistická ročenka Slovenskej republiky. Bratislava: VEDA, 2000, str. 67

Die Größenskala der Betriebe ist mit der Methodik in der EU kompatibel. Sie umfasst drei Betriebsgruppen:

1. 1-49 Mitarbeiter

2. 50-499 Mitarbeiter

3. 500 und mehr Mitarbeiter

Tabelle 2. Größenstruktur des bewerteten Komplexes

\begin{tabular}{|l|c|l|}
\hline Größengruppe & Betriebszahl & $\begin{array}{l}\text { Anteil am Umsatz des } \\
\text { Komplexes }\end{array}$ \\
\hline 1.1-49 Mitarbeiter & 1 & $0,1 \%$ \\
\hline 2.50-499 Mitarbeiter & 38 & $10,9 \%$ \\
\hline 3.500 und mehr Mitarbeiter & 62 & $89,0 \%$ \\
\hline Zusammen & 101 & $100 \%$ \\
\hline
\end{tabular}


Folgende Größenskala liegt dabei zu Grunde (Tabelle 2).

Die durchschnittliche Größe des Betriebes ist in beiden Gruppen $(2,3)$ markant unterschiedlich.

In der Gruppe mit 50-499 Mitarbeitern beträgt sie 0,622 Mrd., und in der Gruppe mit über 500 Mitarbeitern 2,96 Mrd. Es ist also evident, dass es sich im Hinblick auf den gemeinsamen Markteinfluss und die Chancen um unterschiedliche Betriebsgruppen handelt.

\section{Ergebnisse der durchgeführten Umfrage}

\section{A. Internationalisierung des Betriebes}

Wie stark der EU-Markt Einfluss auf den Betrieb als auch seine Chancen nehmen kann, hängt von der Größe und der Internationalisierung des Unternehmens ab. Letzteres haben wir durch ein „System von drei Indikatoren“ geprüft. Die dabei gewonnenen aber auch weitere Daten bestätigen, dass der Anteil am Exporterlös in einzelnen Industriebranchen zwar unterschiedlich, aber dennoch bedeutend ist (Tab. 3, 4).

Tabelle 3. Exportanteil der Unternehmen am gesamten Export der Branche, die mehr als $50 \%$ Anteil am Exporterlös haben

\begin{tabular}{|l|c|}
\hline Branche & Jahr 2001 \\
\hline Maschinenbau & 99,4 \\
\hline Chemie-, Pharmazie- und Kautschukindustrie & 97,3 \\
\hline Metallurgie & 99,5 \\
\hline Energieerzeugung & 86,0 \\
\hline Holzverarbeitungsindustrie & 88,7 \\
\hline Elektroindustrie & 100,0 \\
\hline Konsumgüterindustrie & 100,0 \\
\hline Nahrungsmittelindustrie & - \\
\hline Glasindustrie & 85,7 \\
\hline Produktion von Baumaterialien & 84,8 \\
\hline Druckgewerbe & 100,0 \\
\hline Förderindustrie & 100,0 \\
\hline
\end{tabular}

Die oben genannten Anteile sinken in mehreren Branchen wesentlich, wenn wir die Grenze des Exportsanteils am Erlös auf 75\% steigern (Tab. 4).

Der Einfluss des gemeinsamen EU-Marktes (und Bedarf der Adaptation) soll auch unter dem territorialen Aspekt der Exportorientierung im Hinblick auf den EU-Markt als auch den Markt der V4 Staaten und zukünftiger EU Mitglieder einzelner Betriebe untersucht werden. 
Tabelle 4. Exportanteil der Unternehmen an der gesamten Ausfuhr der Branche, die mehr als $75 \%$ Anteil am Exporterlös haben

\begin{tabular}{|l|c|}
\hline Branche & Jahr 2001 \\
\hline Maschinenbau & 86,9 \\
\hline Chemie-, Pharmazie- und Kautschukindustrie & 26,1 \\
\hline Metallurgie & 92,1 \\
\hline Energieerzeugung & - \\
\hline Holzverarbeitungsindustrie & 27,2 \\
\hline Elektroindustrie & 81,0 \\
\hline Konsumgüterindustrie & 62,6 \\
\hline Nahrungsmittelindustrie & - \\
\hline Glasindustrie & 85,7 \\
\hline Produktion von Baumaterialien & 35,9 \\
\hline Druckgewerbe & - \\
\hline Förderindustrie & 100,0 \\
\hline
\end{tabular}

Wenn man die Tätigkeit einzelner slowakischer Betriebe auf dem EU-Markt betrachtet, können diese Betriebe in drei Gruppen eingeteilt werden (Tab. 5):

Tabelle 5

\begin{tabular}{|l|l|}
\hline Orientierun & \multicolumn{1}{|c|}{ Branche } \\
\hline 1. mit starker Orientierung an der EU & $\begin{array}{l}\text { Maschinenbau } \\
\text { Konsumgüterindustrie } \\
\text { Glasindustrie } \\
\text { Elektrotechnische Industrie }\end{array}$ \\
\hline 2. mit mittlerer Orientierung an der EU & $\begin{array}{l}\text { Chemie-, Pharmazeutische und } \\
\text { Kautschukindustrie } \\
\text { Holzverarbeitungsindustrie } \\
\text { Produktion von Baumaterialien } \\
\text { Druckgewerbe }\end{array}$ \\
\hline 3. mit schwacher Orientierung an der EU & $\begin{array}{l}\text { Nahrungsmittelindustrie } \\
\text { Förderindustrie }\end{array}$ \\
\hline
\end{tabular}

Der Anteil der Branchen mit starker Orientierung am EU-Markt hat in der Exportstruktur des Jahres 2001-40,7 \% des gesamten Exports ausgemacht.

Ein wichtiger Punkt bei der Internationalisierung im Betrieb ist auch die angewandte Zutrittsform zum ausländischen Markt. In der Untersuchung wurden folgende Möglichkeiten in Betracht gezogen:

- indirekter Export (durch fremde Organisationen),

- direkter Export ohne Investitionen (eigene Abteilungen als Bestandteil der Betriebsorganisationsstruktur, z. B. Abteilung für Absatz, Marketingbereich u.a.),

- direkter Export mit Investitionen (repräsentatives Büro, Absatzfiliale im Ausland) 
- Joint Venture im Ausland,

- Tochtergesellschaft im Ausland.

Die Aufteilung der einzelnen Zutrittsformen zum Auslandsmarkt zeichnete sich in der Untersuchung wie folgt ab:

- Indirekter Export

- Direkter Export ohne Investitionen

- Indirekter Export mit Investitionen

- Joint Venture

- Produktionsunternehmen im Ausland
22

71

21

9

10

Die Untersuchung zeigt, dass hauptsächlich einfachere Zutrittsformen zum ausländischen Markt Anwendung finden. Höhere Zutrittformen zum Auslandsmarkt hingegen werden zum Großteil in Betrieben mit über 500 Mitarbeitern angewandt (28 Betriebe dieser Größengruppe erwirtschafteten aber lediglich 49,5 \% der Erlöse der gesamten Erlösen dieser Größengruppe). In der Größengruppe von 50-499 Mitarbeitern werden höhere Zutrittsformen eher weniger bevorzugt - nur vier Betriebe von 38 dieser Größengruppe gehören dazu und machen einen Erlösanteil von 3\% aus.

\section{Tabelle 6}

\begin{tabular}{|l|c|c|}
\hline Stärken & $\begin{array}{c}\text { auf dem Inlandsmarkt } \\
\text { (Anzahl Nennungen) }\end{array}$ & $\begin{array}{c}\text { auf dem EU-Markt } \\
\text { (Anzahl Nennungen) }\end{array}$ \\
\hline Tradition & 3 & 1 \\
\hline Monopolstellung & 2 & 6 \\
\hline eigene F\&E und technologische & 4 & 3 \\
\hline $\begin{array}{l}\text { technische } \\
\text { Stufe }\end{array}$ & 7 & 4 \\
\hline ausreich. Produktionskapazitäten & 4 & 9 \\
\hline Flexibilität & 13 & 5 \\
\hline Billige Arbeitskräfte & 4 & 7 \\
\hline Personal & 8 & 7 \\
\hline Marktkenntnis & 7 & 8 \\
\hline Kundenkenntnis & 8 & 28 \\
\hline Marke & 4 & 3 \\
\hline Qualität & 41 & 1 \\
\hline Sortiment & 10 & 19 \\
\hline Service, Dienstleistungen & 7 & 3 \\
\hline Preis & 25 & 2 \\
\hline Kosten & 5 & 2 \\
\hline Produkt & 13 & \\
\hline gute Finanzlage & 3 & 28 \\
\hline geographische Lage & & \\
\hline ISO & & \\
\hline
\end{tabular}




\section{B. Charakteristik interner und externer Umwelt}

Die Wettbewerbsfähigkeit slowakischer Betriebe ist ein wichtiges Adaptationsinstrument für den Beitritt zum gemeinsamen EU-Markt. Die Betriebe führen in der Untersuchung folgende Stärken an (Tab. 6).

Die Reihenfolge der Stärken aus Sicht der Betriebe zeigt Tabelle 7:

Tabelle 7. Reihenfolge der Stärken des Betriebes

\begin{tabular}{|l|l|}
\hline Inlandsmarkt & EU - Markt \\
\hline Qualiät & Qualiät \\
Preis & Preis \\
Flexibilität & Flexibilität \\
Produkt & Personal \\
Sortiment & \\
\hline
\end{tabular}

Auf beiden Märkten dominieren die Marketinginstrumente Distribution und Kommunikation, die nur wenige Betriebe als Schwächen anführen.

Die Beitrittsgründe slowakischer Betriebe zum EU-Markt sind vielfältig. Es überwiegen aber die Ausnutzung der Produktionskapazitäten sowie die Sättigung des Inlandmarktes und Reduzierung des Risikos (Tab. 8).

Tabelle 8. Anzahl von Zutrittsmotiven zum EU-Markt

\begin{tabular}{|l|c|}
\hline Motiv & Anzahl \\
\hline bessere Ausnutzung der Produktionskapazitäten & 65 \\
auf dem Inlandsmarkt wird kein Wachstum erwartet & 26 \\
der Inlandsmarkt ist gesättigt & 35 \\
Erwerben finanzieller Mittel & 14 \\
Gewinn von ausländischem Know-how & 21 \\
Reduzierung des Risikos & 33 \\
Andere & 6 \\
\hline
\end{tabular}

Entscheidende Informationsquellen über den EU-Markt sind die SOPK (Slowakische Handels- und Industriekammer), sowie die Banken und Handelsvertreter (Tab. 9).

Tabelle 9. Informationsquellen (über den EU-Markt)

\begin{tabular}{|l|c|}
\hline & Menge \\
\hline SOPK & 31 \\
Banken & 30 \\
Handelsvertreter & 24 \\
Persönliche Kontakte & 7 \\
Muttergesellschaft & 6 \\
Ausstellungen und Messen & 6 \\
Andere & 4 \\
\hline
\end{tabular}


Von 101 Unternehmen nutzen nur 30 die verschiedenen Unterstützungsformen des Exports (Tab. 10).

\section{Tabelle 10}

\begin{tabular}{|l|c|}
\hline Unterstützungsform der Ausfuhr & Menge \\
\hline Informations- und Beratungsdienste & 14 \\
Staatsversicherung der Ausfuhr & 13 \\
Finanzierung des Exports & 12 \\
Bewertung der Exportleistungen & 5 \\
\hline
\end{tabular}

Die Einführung der ISO 9000 Normreihe wird als sehr positiv bewertet. Von allen untersuchten Betrieben ist diese in $68 \%$ der Betriebe eingeführt worden. Ohne Einführung dieser Normen ist es für ein exportorientiertes Unternehmen unmöglich, seinen Erfolg auszubauen. Die Einführung der Normen ISO 14000 geht langsamer voran. Dabei ist zu bemerken, dass ein starker Einfluss der Branchen eine Rolle spielt.

Die Ausnutzung von Adaptationsstrategien steht im „Einklang“ (was sehr erfreulich ist) mit den anderen Ergebnissen der Teilnehmeruntersuchung (insbesondere bezüglich der Beitrittsmotive zum EU-Markt und der Zutrittsformen zum ausländischen Markt. (Tab. 11). Die häufigsten Strategien sind Kooperation, Expansion und Diversifikation.

Tabelle 11. Strategien die beim Beitritt zum EU-Markt angewendet werden

\begin{tabular}{|l|c|}
\hline Ausgewählte Strategien & Häufigkeit \\
\hline Kooperation & 45 \\
Diversifikation & 26 \\
Akquisition & 8 \\
Fusion & 7 \\
Lückenstrategie & 8 \\
Expansion & 27 \\
\hline
\end{tabular}

Die untersuchten Unternehmen stehen dem Beitritt der SR zur EU insgesamt sehr optimistisch gegenüber. Auf die Frage, ob sie einen positiven Einfluss vom Beitritt in die EU erwarten, antworten 80 Betriebe mit ja $(79,2 \%$ der gesamten Anzahl), nein antworten 9 Betriebe $(8,8 \%$ aus der gesamten Zahl), und 12 Unternehmen antworten ,ich weiß nicht“ (dies bedeutet 11,9\%). Weniger optimistisch sind die betrieblichen Erwartungen in Bezug auf Ertrag und Gewinn (Tab. 11), wo geringe und durchschnittliche Unternehmenserwartungen überwiegen, mit zusammen $74,3 \%$ der gesamten Unternehmenszahl des untersuchten Komplexes.

Einzelne slowakische Betriebe erwarten vom Beitritt zum gemeinsamen EUMarkt eine Erhöhung der Absatzmöglichkeiten und die Abschaffung von (Zoll-, Verwaltungs- und anderen) Hindernissen. 
Tabelle 12. Erwartungen der Betriebe

\begin{tabular}{|l|c|c|}
\hline Erwartungen der Betriebe & Zahl der Betriebe & \% in den Betrieben \\
\hline Geringe & 23 & 22,8 \\
\hline Durchschnittliche & 52 & 51,5 \\
\hline Große & 26 & 25,7 \\
\hline Zusammen & 101 & 100,0 \\
\hline
\end{tabular}

Einzelne slowakische Betriebe erwarten vom Beitritt zum gemeinsamen EUMarkt eine Erhöhung der Absatzmöglichkeiten und die Abschaffung von (Zoll-, Verwaltungs- und anderen) Hindernissen.

Auf die Frage, ob der Einfluss des Gemeinsamen Marktes auf den eigenen Betrieb spürbar ist, ergibt sich folgendes Ergebnis: 70 Betriebe antworten mit ,ja“, 22 antworten „,nein“ und neun Betriebe können den Einfluss des gemeinsamen Marktes nicht bewerten. Die Mehrheit der Betriebe schätzt den Einfluss des gemeinsamen Marktes als durchschnittlich bis gering ein, und unterschätzt dadurch den Einfluss (63 Betriebe, das sind 62,4 \% der Gesamtzahl der untersuchten Betriebe). Gleichfalls ist die Mehrheit der Betriebe sehr optimistisch und erwartet vom Beitritt zum Gemeinsamen EU-Markt Chancen das sind 61 Betriebe (60,4 \% der Betriebsgesamtzahl), 12 Betriebe sehen Chancen und auch Risiken, und der Rest, 28 Betriebe, geben nur an, Risiken zu sehen. Berechtigte Skepsis haben Betriebe der Nahrungsmittelindustrie.

Auch die an der Lösung der Forschungsaufgabe beteiligten Facharbeiter beurteilen die Einflussmöglichkeit, Chancen und Risiken des gemeinsamen Marktes,. Die Ergebnisse dieser Bewertung zeigen, dass 32 Betriebe der Größengruppe über 500 Mitarbeiter (Gesamtzahl 62) den Einfluss und die Chancen, „richtig“ einschätzen. Der Anteil der Erlöse dieser Betriebe (32) am gesamten Erlös des untersuchten Komplexes (101 Betriebe) beträgt 48,9 \% (durchschnittliche Größe 3,2 Mrd.). Es geht also hauptsächlich um Betriebe, deren Erlöse höher als der durchschnittliche Erlös dieser Gruppe sind.

In der Größengruppe 50-499 Mitarbeiter beurteilen 17 Betriebe (von 37) den Einfluss des gemeinsamen Marktes und die Chancen als ,richtig“. Der Erlösanteil der ,richtig bewertenden“ Betriebe am gesamten Erlös des untersuchten Komplexes macht $56,4 \%$ aus.

Insgesamt kann man feststellen, dass 49 Betriebe (von 101) den Einfluss des gemeinsamen Marktes und auch mögliche Chancen ,richtig“ beurteilten, wobei der Anteil an den Erlösen des untersuchten Komplexes 49,8 \% ausmacht.

\section{Strategische Betriebsentwicklung}

Ein Auslandsinvestor stellt in der Regel ein gutes Adaptionsinstrument für den Beitritt zum gemeinsamen EU-Markt dar. Im beurteilten Unternehmenskomplex (101) war der ausländische Investor in 17 Unternehmen der Größengruppe 50499 Mitarbeiter, und in 22 Unternehmen der Größengruppe über 500 
Mitarbeiter involviert (nur selten handelt es sich um Investoren aus Staaten außerhalb der EU). Die Erlöse der Unternehmen mit Beteiligung ausländischer Investoren nehmen folgenden Anteil an den Gesamterlösen einzelner Größengruppen ein:

- Größengruppe 50-499 Mitarbeiter 56,4 \%,

- Größengruppe über 500 Mitarbeiter 47,3\%.

Der Anteil der Erlöse von 39 Unternehmen mit ausländischer Beteiligung an den Gesamterlösen des untersuchten Komplexes beträgt fast die Hälfte, 48,8 \%.

Die Motive der Auslandsinvestoren sind vor allem Marktmotive (Erweiterung des Marktes, Sicherstellung des bestehenden Marktes) und niedrigere Lohnkosten (Tab. 13).

Tabelle 13. Motive der Auslandsinvestoren

\begin{tabular}{|l|c|}
\hline Motiv & Häufigkeit \\
\hline Gewinnung neuer Märkte & 19 \\
Sicherstellung bestehender Märkte & 12 \\
Niedrigere Lohnkosten & 20 \\
Niedrigere Steuerbelastung & 1 \\
Bessere Beschaffungs- und Versorgungsmöglichkeiten & 2 \\
Staatliche Unterstützung der Investitionen & -- \\
Erwartungen eines höheren Gewinns & 7 \\
Andere & 2 \\
\hline
\end{tabular}

Eine Überraschung ist der „Nullwert“ bei der staatlichen Unterstützung von Investitionen. Der Beitrag für die Unternehmen bestand hauptsächlich aus Technologien, Manager- und Marketing-Knowhow.

Positiv kann man auch die Zufriedenheit der Auslandsinvestoren - 34 von 39 bewerten.

\section{Schlussfolgerungen}

Die slowakische Industrie ist durch hohe Export- und Erlöskonzentration gekennzeichnet. Dies spiegelt sich in den Ergebnissen jeder Untersuchung wieder.

Die Internationalisierung der Unternehmen ist in der slowakischen Industrie auch von der Branchenzugehörigkeit abhängig, da vor allem Unternehmen des Maschinenbaus und der Maschinen-, Verbrauchs-, Glas- und Elektronikindustrie einen hohen Exportanteil an Erlösen aufweisen.

Aus Sicht der Adaptationsinstrumente hat die territoriale Orientierung des Exports - insbesondere in die EU - für den Beitritt zum gemeinsamen Markt Bedeutung. Auch hier spielt die Branchenzugehörigkeit eine Rolle. 
Zum Adaptationsinstrument wird auch die angewandte Zutrittsform zum Auslandsmarkt. Die Unternehmen der slowakischen Industrie nutzen hauptsächlich niedrigere Zutrittsformen zum Auslandsmarkt - indirekter Export und direkter Export ohne Investitionen. Höhere Zutrittsformen zum ausländischen Markt verwenden vor allem Unternehmen der Größengruppe über 500 Mitarbeiter (jedoch weniger als erwartet), und dabei eher von den größeren Unternehmen dieser Gruppe (nach Erlösen). Bedenklich ist, dass höhere Zutrittsformen zum Auslandsmarkt in der Größengruppe 50-499 Mitarbeiter kaum angewandt werden.

Die Beobachtung der internen und externen Umgebung ist (teilweise) mit der Beurteilung der Konkurrenzfähigkeit am gemeinsamen EU-Markt verbunden, und führte zu diesen Schlussfolgerungen:

- Die entscheidenden Stärken der slowakischen Unternehmen sind hauptsächlich Qualität und ein niedriges Preisniveau, wobei angemessene Qualität zum ,guten“ Preis nicht im Widerspruch steht.

- Der niedrige Preis könnte in Zukunft besonders für Unternehmen aus Branchen mit hohen materiellem und energetischem Aufwand ein mögliches Risiko darstellen.

- Als Schwächen treten die Marketinginstrumente Distribution und Kommunikation hervor (sie erscheinen auch in anderen Untersuchungen).

- Entscheidende Motive der Industriebetriebe für den Einstieg in den gemeinsamen EU-Markt sind hauptsächlich das Bestreben, die Produktionskapazitäten voll auszunutzen und die Tatsache, dass der Inlandsmarkt gesättigt ist und dadurch das Risiko gestreut wird.

- Die Anwendung der ISO 9000 ist in den Betrieben gut angenommen von insgesamt 61 Unternehmen haben in der Größengruppe über 500 Mitarbeiter nur 8 Unternehmen keine ISO 9000 (vorwiegend in der Verbrauchs- und Glasindustrie). In der Größengruppe 50-499 Mitarbeiter ist die Vertretung der ISO 9000 niedriger, In der Größengruppe 50-499 Mitarbeiter hat sich die Anwendung der ISO 9000 weniger durchgesetzt.

- Überraschend ist die geringe Anwendung aller beliebigen Unterstützungsformen der Ausfuhr.

- Als entscheidende Informationsquellen über den Gemeinsamen EUMarkt werden SOPK, Banken und Handelsvertreter angegeben.

- Als positiv zu bewerten ist, dass die ausgewählten Adaptionsstrategien im Einklang mit den Antworten auf mehrere andere Fragen im Einklang stehen. Im Beitrittsprozess scheinen die ausgewählten Adaptionsstrategien - Kooperation, Diversifikation und Expansion (für Großunternehmen der Größengruppe über 500 Mitarbeiter) vielversprechend zu sein. 
- Auslandsinvestoren können ein wichtiges Adaptationsinstrument sein, besonders wenn sie aus der EU stammen. Als positiv kann man die ausgeglichene Unternehmenszahl mit ausländischer Beteiligung in beiden Größengruppen bewerten. Das ist auch Ausdruck der Zufriedenheit der Auslandsinvestoren mit ihrer Tätigkeit in der SR. Bedenklich sind jedoch die Motive der Auslandsinvestoren, auch die fehlende staatliche Unterstützung von Auslandsinvestitionen.

- Die hohen Erwartungen slowakischer Industrieunternehmen an den Beitritt zur EU sind für die an der Untersuchung beteiligten Fachkräfte keine Überraschung (sie tauchen auch in anderen Untersuchungen auf). Dennoch ist nicht offensichtlich, dass dieser Optimismus auf einer Unterschätzung des Einflusses des EU-Marktes auf die Betriebe (wie auch die Untersuchung des betrachteten Komplexes zeigte), oder auf mangelnder Differenzierung zwischen dem Beitritt der SR zur EU und den Ertrags - und Gewinnmöglichkeiten einzelner Unternehmen, basiert. Ebenso überbewertet sind auch die erwarteten Chancen. Im untersuchten Komplex kann man die Gesamtsituation folgendermaßen bewerten. Die Unternehmen, die $50 \%$ der Erlöse am Gesamtkomplex aufweisen, bewerten ihre Situation als ,richtig“ (es handelt sich besonders um die Unternehmen mit einem Personalstand von über 500 und starker territorialer Orientierung auf die EU).

Aus den Schlussfolgerungen ergeben sich folgende Verallgemeinerungen der Untersuchungsergebnisse:

- Größere Industriebetriebe (mit mehr als 500 Mitarbeitern und mit einem Durchschnittserlös von über 2,9 Mrd. SK), z.B. Betriebe des Maschinenbaus, sowie der elektrotechnischen, Glas- und Konsumgüterindustrie sind in der Slowakei auf den Beitritt besser vorbereitet, als Kleinbetriebe.

- Aus der Untersuchung geht hervor, dass in diesen Betrieben auch eine Übereinstimmung zwischen den Erwartungen und der realen Bereitschaft entsteht. Dasselbe lässt sich auch bei den Betrieben mit Beteiligung der besonders aus den EU - Ländern stammenden ausländischen Investoren beobachten.

- Gleichzeitig zeigt sich, dass im Beitrittsprozess nach wie vor größere Aufmerksamkeit den mittelständischen Betrieben, mit Mitarbeiterzahl 50 -499 zu widmen ist.

\section{Literaturverzeichnis}

Majtán, M. (2001): Manažment (Management), Bratislava: Vydavatel'stvo Ekonóm.

Majtán, M. (2001): Projektový manažment v podnikovej praxi. In: Zborník zmedzinárodnej konferencie „Česká ekonomika na přelomu tisíciletí“. Brno: Masarykova univerzita. 
Porter, M. (1990): The Competitive Advantage of Nations. New York: The Free Press.

Sršňová, J. (2002): Odvetvové a podnikové aspekty slovenského vývozu. In: Biatec, roč. 5, 24.

Sršňová, J. (2002): Teórie a prax zahraničných investícií. In: Ekonomické rozhl’ady, roč. XXXL, 3, 362-370.

Sršňová, J. (2002): Vybrané charakteristiky zahraničných investícií v SR. In: Biatec, roč. 10, 2, 2-5.

Sýkora, P. (2000): Európska integrácia. Bratislava: Centrum európskych štúdií UK.

Šáková, B. (2000): Slovensko a Európska únia - Od susedstva k členstvu. Bratislava: Sprint.

Š́bl, D. (1998): Európska únia a Slovensko. Brastislava: Sprint, 1998.

Tang, H. (2000): Winners and Losers of EU Integration. Washington, D. C.: The World Rank 